weeks' duration or more or where fibrotic sclerosis. Blood urea and electrolytes and urine reactions appeared to be marked. (2) Diaze- analysis normal.

pam $5 \mathrm{mg}$ three times daily. (3) Antiinflammatory drugs (phenylbutazone). The effect of the diagnostic injection of lignocaine lasted a few hours to a few days and was rarely permanent. Early return of pain was a poor prognostic sign. Injections of lignocaine and $1 \%$ alcohol once a week with medication cleared $60 \%$ of the symptoms in six weeks and $90 \%$ in 12 weeks. Headaches were often devastating initially but resolved more rapidly than the neck and back symptoms.

In 26 patients serious bone and brain injuries were identified; two of these died and six were operated on. Twenty patients showed objective signs of lumbar intervertebral disc rupture with nerve root compression, but only three were operated on, as their symptoms resolved over a period with conservative management by injection. -I am, etc.,

\section{Raphakl Cillento}

Northville State Hospital,

Northville, Michigan

\section{Mixed Connective Tissue Disease}

SIR,-Your leading article (11 November, $p$. 315) shows that the delineation of "mixed connective tissue disease"l as a distinct entity appears at present to be rather more than an exercise in nosology. It remains to be seen, however, whether or not further experience will blur the edges of the currently fairly clear-cut situation.

Among the classical connective tissue diseases, progressive systemic sclerosis showing features of systemic lupus erythematosus (S.L.E.) $)^{2}$ can provide diagnostic problems. In 1965 we $^{3}$ drew attention to the occurrence in S.L.E. of small stiff lungs giving rise to severe dyspnoea. It is now apparent that "lupus lung" is not specific for S.L.E. but can occur in systemic sclerosis, as the following case report shows.

A 55-year-old Italian housewife presented in October 1968 with a two-month history of bilateral Raynaud's phenomenon and severe ischaemia of a finger-tip. She was known to have a hiatus hernia and had taken amphetamines (Durophet) for many years as an anorectic In December 1968 she was admitted to another hospital with pleurisy, fever, and consolidation of the right lower lobe of the lung, which cleared after 10 days. In January 1969 she complained of severe breathlessness on slight exertion. Her left diaphragm was now elevated and later a linear shadow appeared at the left base. Her respiratory function was impaired : FEV $_{1}$ 1.0 1., FVC 1.5 1. A lung scan was suggestive of multiple pulmonary emboli.

In May 1969 she was severely dyspnoeic at rest and unable to climb stairs. There was pain in the lumbar and shoulder regions, especially on deep breathing, together with severe Raynaud's phenomenon. She later brought up undigested food and gave a history of dysphagia for some months. On examination her fingers losked sclerodermatous. Heart and lungs were clinically normal, blood pressure $130 / 80 \mathrm{~mm} \mathrm{Hg}$. There was no evidence of pulmonary hypertension. Investigations : $\mathrm{Hb} 14.9 \mathrm{~g} / 100 \mathrm{ml}$ W.B.C. $6,800 \mathrm{~mm}^{3}$, differential normal, E.S.R. $10-20 \mathrm{~mm}$ in $1 \mathrm{hr}$. Tests for antinuclear factor, L.E. cells, and rheumatoid factor (latex) negative. Serum flocculation tests strongly positive, Chest $x$-ray showed bilateral high diaphragms with linear basal shadows. B.C.G., right heart catheterization, and pulmonary angiogram normal FEV 10.51 . FVC 0.91 . Skin biopsy not diagnostic. Barium swallow suggestive of systemic
She was treated initially with warfarin and later with prednisone, on which she felt less breathless. Her respiratory function tests improved a little. She had several episodes of nocturnal pulmonary aspiration, in one of which she had a cardiac arrest and died in August 1969.

Postmortem showed the classical histological changes of systemic sclerosis in the skin and oesophagus. The lungs showed changes of chronic bronchitis but no evidence of pulmonary fibrosis, embolism, infarction, or arteritis. The heart, pericardium, and pleural spaces were

This patient had a fulminating disease, dying of progressive systemic sclerosis less than 12 months after her initial symptoms. The chest picture, with severe breathlessness, gross restrictive impairment of ventilation, a radiological picture resembling pulmonary infarction, and the absence of specific lung pathology was identical to that found in S.L.E. ${ }^{3}$ There are thus at least two causes for dyspnoea in progressive systemic sclerosis in addition to the well-recognized pulmonary fibrosis. One is pulmonary arteritis," the other "lupus lung."-I am, etc.,

\section{B. I. HOFFBRAND}

Whittington Hospital,

1 Sharp, G. C., Irvin, W. S., Tan, E. M., Gould, of G., and Holman, $H$. . $R$.

Dubois, B. L., Chandor, S.; Friou, G. J., and Bischel, M., Medicine. 1971, 50.199. .
Hofitorand B. I., and Beck. B. R., British MediHoftrand B. I., and Beck.
cat Jov-nal, 1965,1 , 1273 .
Case Records of the Missach Pitial, New England Journal of Medicine, 1972,
286, 91.

\section{Poisoning Treatment Centres}

SIR, - We were interested to read the article by Dr. Peter Kennedy, (4 November, p. 255) comparing the prognosis in parasuicides admitted to a poisoning treatment centre with that in those treated by a psychiatrist as an outpatient or by a general practitioner.

In a recent paper ${ }^{1}$ we described the medical aspects of acute poisoning as seen in a general medical unit and showed that the results compared favourably with those obtained in more sophisticated centres. The social and environmental aspects of acute poisoning in the same area have also been described.2 Of 131 patients admitted to our unit during 1970 after attempted suicide, only $11(8.4 \%)$ had a further episode during 1970-1. Our recurrence rate is thus lower than Dr. Kennedy's figure of $13 \%$ despite the fact that we have included all our patients admitted in 1970 (not merely those admitted for the first time), and we have included recurrences in these patients until recurrence rate in a general medical unit without immediate psychiatric cover suggests that Dr. Kennedy is justified in being cautious about his conclusion that special units like the one in Edinburgh should be set up in other regions.

Dr. Kennedy compared only the results of management in a regional poisoning treatment centre with outpatient psychiatric care and general practitioner care. It would have been more reasonable to compare treatment in general hospitals (where most patients with self-poisoning are still treated) with treatment at home or in a sophisticated centre. It is possible that admission to hosthe end of 1971 rather than 1970. This low pital, by temporarily withdrawing the patient from a hostile environment, may be therapeutic quite apart from psychiatric intervention. In contrast to Dr. Kennedy, we concluded" that it was "possible to treat successfully the vast majority of acutely poisoned patients in any general medical unit." This statement was concerned primarily with medical treatment, but we feel it is also applicable to psychiatric intervention.

We would continue to support the concept of a limited number of Regional Poisoning Treatment Centres as areas where sophisticated toxicological and epidemiological studies can be performed, but there seems little justification for the full implementation of the Hill Report. ${ }^{3}$ - We are, etc.,

\section{A. A. H. LAwSON} I. Mrtcheil

Milesmark Hospital,

\section{Dunfermline}

1 Lawson, A. A. H., and Mitchell, I., British
Medical fournal, 1972, 4, 153. H. Community
2 Mitchell, I, and Lawson, A. A. H., Commuits
Medicine, In press.
3 Department of Health and Social Security, Cen-
tral and Scottish Health Services Councils,
Hospital Treatment of Acute Poisoning, Lon-
don, H.M.S.O., 1968.

\section{Hexpes Simplex Encephalitis}

SIR,-In the interesting and important paper by Dr. A. D. Dayan and others (18 November, p. 405) there is a reference to our work ${ }^{1}$ which is flattering but inaccurate. In our series of electroencephalographic findings in herpes simplex encephalitis (which is the largest series in the literature) we found that the electroencephalogram reflects the severity of the illness and the site of maximum damage rather than the pathology. We also pointed out that similar changes may be seen in cerebrovascular disease, raised intracranial pressure, and other conditions. The electroencephalogram in herpes simplex encephalitis is only "diagnostic" in the sense that it may help to make the diagnosis, but we would like to stress that the electroencephalographic findings are in no way pathognomonic.-We are, etc.,

L. S. ILLIS

FLORA M. TAYLOR

Wessex Neurological Centre,

Southampton General Hospital, Southampton

1 Illis, L. S., and Taylor, F. M., Lancet, 1972,

\section{Causes of Failure in Antibiotic Treatment}

SIR,-Professor L. P. Garrod (25 November, p. 473) points out that Mr. D. S. Tindal and $P$ did not say how many wound infections in a controlled series of patients given a single parenteral dose of ampicillin were due to penicillin-resistant staphylococci.

We were concerned only with the clinical control of wound infections, and in many cases pus swabs were not taken. A few swabs grew more than one organism. Altogether, 164 patients were given ampicillin and there were 22 infections. In 12 cases no pus swab was taken and in four it was sterile. Five patients were infected with staphylococci insensitive to ampicillin and two had coliform organisms, also insensitive to ampicillin. In the control series of 185 patients not given ampicillin, 25 developed a wound infection. In 12 cases no 